\title{
Autonomic nervous system function in Huntington's disease
}

\author{
J Andrich, T Schmitz, C Saft, T Postert, P Kraus, J T Epplen, H Przuntek, M W Agelink
}

J Neurol Neurosurg Psychiatry 2002;72:726-731

See end of article for authors' affiliations

.....................

Correspondence to: Dr M W Agelink, Department of Biological

Psychiatry and

Neuroscience, The

Evanglical Clinics

Gelsenkirchen,

Ruhr-University of Bochum

Munckelstr 27, D-45879

Gelsenkirchen, Germany

Received 24 May 2001

In final revised form

19 November 2001

Accepted

13 December 2001

\begin{abstract}
Objective: To investigate whether Huntington's disease (HD) affects autonomic nervous system (ANS) functioning.

Methods: Twenty patients with HD who had positive genetic test results underwent standardised ANS function tests including sympathetic skin responses (SSRs) of the hands and feet, measurements of heart rate variability (HRV), both during five minutes of resting and deep respiration, and an orthostatic blood pressure test. Patients were classified according to the motor subscale of the unified Huntington's disease rating scale (UHDRS; mean (SD) score 26.4 (13.6)) and divided into two subgroups: UHDRS <25 points (early stages, E-HD) and UHDRS $\geqslant 25$ points (mid stages, M-HD). Autonomic indices were compared with those obtained for a group of well matched healthy controls $(n=60)$.

Results: Overall, patients showed lower HRV indices than controls. Multivariate analysis with the independent factor of "group" (controls, E-HD, M-HD) showed a significant group effect on both the high frequency power $(F=4.32, p=0.017)$ and the coefficient of variation $(F=4.23, p=0.018)$, indicating a significant reduction in vagal modulation in the M-HD group. There was a shift in autonomic neurocardiac balance towards sympathetic predominance in the $M-H D$ group compared with controls ( $F=2.89$, $p=0.062$ ). Moreover, we found an inverse correlation between the severity of clinical HD symptoms (assessed by the UHDRS) and the modulation of cardiovagal activity $(p=0.028)$. Vagal dysregulation was present in two patients; one of them also showed a pathological blood pressure test and a latency prolongation in the SSRs of the hands. Two other patients had pathologically reduced SSR amplitudes. Only patients of the M-HD group were affected.

Conclusion: Autonomic dysfunction is present even in the middle stages of HD and affects both the sympathetic and parasympathetic branch of the ANS.
\end{abstract}

n Huntington's disease (HD), the protein huntingtin is enlarged because of an extended CAG repeat chain (more than 36 units) on the short arm of chromosome 4. The defect of this protein promotes the apoptotic cell death of neurones in various brain regions, especially the stem ganglia (particularly the caudate nucleus). However, diffuse neuronal degeneration also occurs in other areas of the brain such as the frontal cortex. The result of this nerve cell loss is a clinical triad of motor disturbances (mostly hyperkinesis, subcortical dementia, and psychiatric symptoms). As huntingtin is expressed in virtually all body cells to a varying extent, other organ systems - for example, skeletal muscles-may also be affected by the degenerative process.

Only scant and inconsistent data have as yet been reported on whether HD correlates with disruptions in the function of the autonomic nervous system (ANS). ${ }^{2-5}$ Both the presence $e^{5}$ and absence ${ }^{4}$ of parasympathetic dysfunction have been postulated in patients with HD. With regard to the sympathetic nervous system, defects in the central autonomic network-for example, the hypothalamus and its connection to the neocortex, the limbic system, brainstem, and spinal cord-have consistently been postulated. ${ }^{35}$ However, considerable discrepancies in the results of autonomic tests were evident despite this apparent consistency; as an example, normal sympathetic skin response (SSR) findings were produced in one study, whereas in another there were prolongations in latency, amplitude reductions, and even lacking potentials. ${ }^{5}$ These sometimes highly divergent findings may be related to the considerable methodological differences between the studies quoted. Differences concerned not only the selection of autonomic functional tests-for example, measurement of heart rate variability (HRV) as an expression of parasympathetic regulation using various measurement procedures and mathematical statistical analyses-but also the nature and composition of the patient samples-for example, age and size of the samples, stage of HD disease, concomitant treatment with various drugs. The study presented here accounts for the various methodological aspects by: $(a)$ genetic testing of the diagnosis of HD in all cases; $(b)$ selecting patients with HD by considering the results of extensive auxiliary examinations and definition of strict exclusion criteria; (c) standardised recording of the severity of HD symptoms using the unified Huntington's disease rating scale (UHDRS); $(d)$ comparing patient data with those obtained from a large group of healthy controls $(\mathrm{n}=60) ;(e)$ applying standardised autonomic functional tests involving computerised measurements of HRV. To our knowledge, the last mentioned procedure has not as yet been applied to patients with HD. Unlike conventional HRV bedside tests, as already used in other studies, ${ }^{3-5}$ the standardised five minute HRV resting study in particular allows an adequate, quantitative estimation of autonomic neurocardiac function under steady state conditions in a way that is largely independent of patient cooperation. ${ }^{6}$

\section{METHODS}

\section{Study population}

Twenty patients with HD were included in the study. Written informed consent was obtained from patients after a detailed explanation of the procedures. All patients had positive genetic

Abbreviations: HD, Huntington's disease; ANS, autonomic nervous system; UHDRS, unified Huntington's disease rating scale; HRV, heart rate variability; ECG, electrocardiogram; SSR, sympathetic skin response; $L F$, low frequency; HF, high frequency 
test results, with CAG blocks of more than 36 triplets (mean (SD) of 45.9 (4.0), range 42-57). All of them were symptomatic for HD at an early or middle stage with a mean (SD) UHDRS of 26.4 points (13.6) ranging from 5 to 46 points and with a mean (SD) time between disease onset and participation in the study of 3.8 (2.5) years (range 0.5-9). Exclusion criteria included the presence of diseases or conditions known to influence regulation of the ANS; examples include medical disorders (cardiac rhythm disorders, cardiac insufficiency, arterial hypertension requiring treatment, chronic obstructive pulmonary disease treated with bronchodilatators, renal diseases, liver diseases), endocrinological disorders (thyroid dysfunction, diabetes mellitus), malnutrition, underweight (body weight 15\% lower than the ideal weight), or drug abuse (although patients with nicotine abuse were not excluded). Patients who presented signs of other neurological disorders apart from HD, in particular signs of a peripheral neuropathy, were not included. None of the patients were suffering from a psychiatric disease, such as major depression, anxiety disorder, or schizophrenia according to DSM-III-R criteria. For patients taking drugs with known anticholinergic effects, a wash out phase of at least 48 hours is recommended before autonomic functional tests are carried out. ${ }^{7}$ Six of the patients had previously taken drugs with known anticholinergic properties-for example, neuroleptics-which were discontinued in all cases at least three days (range 3-8) before study entry. No patient had received any psychiatric drugs with very long half lives, such as depot neuroleptics and fluoxetine.

Normal values for HRV measurement procedures were obtained from 60 healthy probands. They were medical and non-medical employees of the hospital clinics in Gelsenkirchen and Düsseldorf as well as medical and non-medical students of the Ruhr-University Bochum and their family members. None of the controls were receiving regular drug treatment. Only the use of oral contraceptives and an occasional intake of non-steroidal anti-inflammatory drugs such as acetylsalicylate or paracetamol were permitted. Patients and controls were matched according to their socioeconomic background. There were no significant differences in basic data between patients and controls (table 1).

\section{Study programme}

A resting electrocardiogram (ECG), a thoracic $x$ ray examination, and routine laboratory tests were performed in all cases. Before study entry, all patients underwent a comprehensive psychiatric investigation by an experienced psychiatrist (MA). A standardised questionnaire was used to screen for complaints suggestive of autonomic dysfunction. The neurologist carrying out the neurological examination was blinded with respect to the results of the autonomic tests.

\section{UHDRS}

Motor disturbances were evaluated by using the motor function subscale of the UHDRS as recommended by the Huntington Study Group ${ }^{8}$ with a range of $0-124$ points. As there is no information about autonomous dysfunction in presymptomatic patients with HD and because of the difficulties associated with SSR or HRV measurement in severely hyperkinetic probands, no patient with less than 5 points

Table 1 Basic details of the study population

\begin{tabular}{|c|c|c|c|}
\hline & $\begin{array}{l}\text { Controls } \\
(n=60)\end{array}$ & $\begin{array}{l}\text { Patients } \\
(n=20)\end{array}$ & Statistical analysis \\
\hline Age (years) & 40.0 (10.1) & $39.9(10.3)$ & Mann-Whitney test \\
\hline Age range & $25-69$ & $25-65$ & Not significant \\
\hline Male & $22(36.7 \%)$ & $7(35 \%)$ & $\chi^{2}$ test \\
\hline Female & 38 (63.3\%) & $13(65 \%)$ & Not significant \\
\hline Smokers & $20(33.3 \%)$ & $6(30.0 \%)$ & $\chi^{2}$ test \\
\hline Non-smokers & $40(66.7 \%)$ & $14(70.0 \%)$ & not significant \\
\hline
\end{tabular}

Age is given as mean (SD) (asymptomatic) or more than 50 points (medium to severely disabled) on the UHDRS was included. The patients were divided into two groups: those with $<25$ points (very early stage of the disease; E-HD group) and those with $\geqslant 25$ points (mid stage of the disease; M-HD). The motor score of the UHDRS includes eye movements, hyperkinesia and dyskinesia, gait, muscle tone, and other movement tests; this score correlates highly with the deterioration of the basal ganglia in HD.

\section{Five minute resting HRV}

The evaluation and execution of HRV measurements was based on previously published experimental procedures. ${ }^{69^{10}}$ To analyse HRV, we used the Neuro-diag software program $(\mathrm{H}$ Lambeck, Munich, Germany). With this system, a fast machine code algorithm recognises the typical form of the QRS complexes from a set of parameters characterising the time course of the signal: steepness of the ascending and descending slopes, rise time, and amplitude. The optimal setting of these parameters is evaluated from individual ECGs during a learning phase that is automatically activated before the actual measurement phase. During measurement, the optimal settings of the QRS recognition parameters are continuously adjusted to the changes in ECG waveforms observed-for example, with deep breathing. To control the reliability of QRS detection, the ECG signal is continuously displayed on a computer screen and the correctly recognised QRS complexes are automatically marked on the monitor. The R-R intervals were measured to an accuracy of 1 millisecond. The artifact-free, digitised signal was stored on a PC for later analysis.

The examination was started after the subjects had rested for 10 minutes in a relaxing and comfortably temperate room on the examination bench. They were asked to breath regularly and calmly and to move as little as possible. Even during the resting phase before the start of the actual measurement period, the study leader was asked to supervise the probands closely, and, if necessary, provide instructions on breathing technique and relaxation. In this way it could be guaranteed that the respiratory rhythm and depth of the probands remained largely constant during the measurement period (breath frequency approximately $0.2 \mathrm{~Hz}$; corresponding to 12 breaths per minute). If these rules are followed, one need not adhere to metronomic breathing during the five minute resting study of HRV, as our own experience and the results of other groups have shown. ${ }^{11}$

The resting heart rate was defined as the average heart rate during the five minute resting examination. Time domain variables included the variation coefficient $(\mathrm{CVr})$, defined as the ratio of the standard deviation of the R-R intervals divided by the average duration of the R-R intervals, and the root mean square of successive differences (RMSSDr); both represent parasympathetic modulation. ${ }^{6}{ }^{10}$ Data used for calculating the spectral powers were the artifact-free R-R intervals registered over a period of five minutes at a temporal resolution of 1 millisecond. The interval series was converted using a mathematical algorithm $^{12}$ into a discrete signal of 1024 levels (computed at equidistant sampling points 290 milliseconds apart). The resulting power spectrum was calculated using a fast Fourier transformation, whereby three frequency bands were automatically separated: the very low frequency band $(0.003-0.04 \mathrm{~Hz})$, low frequency band (LF; 0.04-0.15 Hz), and high frequency band (HF; $0.15-0.4 \mathrm{~Hz}$ ). Owing to the short five minute analysis period, a conclusive interpretation of the very low frequency power was not possible, ${ }^{6}$ and for this reason we restricted the evaluation to LF and HF powers. The HF band coincides with the respiratory frequency and reflects mainly respiration linked variations in heart rate resulting from centrally mediated cardiac vagal control. ${ }^{6}$ The LF band is probably associated with both parasympathetic and sympathetic activity and represents a reflection of the baroreflex response. ${ }^{6}$ The LF/HF ratio is considered by most, ${ }^{13-15}$ although not all, ${ }^{16}$ investigators to be a measure of the sympathetic-parasympathetic balance. 


\section{Deep breathing test}

Subjects were instructed to take six deep breaths per minute (six second inspiration, four second expiration). The respiratory cycles were depicted on a computer monitor by rising and falling columns. The resulting changes in heart rate, the breath dependent amplitudes of the R-R deflections, and properly recognised QRS complexes were also registered. The variation coefficient $(\mathrm{CVd})$ and the root mean square of successive differences (RMSSDd) were calculated from at least 100 artifact-free R-R intervals. ${ }^{910}$ A geometrically constructed variable, the mean circular resultant, was calculated by vector analysis. ${ }^{17}$ All three HRV indices depend mainly on parasympathetic regulation.

\section{Blood pressure to standing}

This test was used to evaluate sympathetic regulation. After their resting blood pressure had been measured (defined as the mean of three consecutive measurements made at two minute intervals) in the supine position, the patients were asked to stand up quickly. Blood pressure was then measured twice (immediately after standing up and one minute later), and the differences in systolic and diastolic blood pressure were determined for both occasions.

\section{SSR and nerve conduction velocity}

SSRs from both hands and feet were investigated using standard methods. ${ }^{18}$ Briefly, standard disc electrodes $(10 \mathrm{~mm})$ were attached to the palms and soles as active electrodes and to the dorsum of the hands and feet as reference electrodes. An electromyograph (Dantec; Medtronic GmbH, Düsseldorf, Germany) was used to create the stimulus and monitor the potentials. Stimulation was performed twice in the median nerve (right thumb, after exclusion of carpal tunnel syndrome) and twice supratrochlearly by using a square wave pulse of 0.2 milliseconds duration and $25 \mathrm{~mA}$ intensity. The stimuli were given at irregular intervals of more than five minutes to prevent habituation. The best of the four records was selected as the representative SSR. The latency (in ms) between the onset of the stimulus and the first negative deflection was measured. Amplitude (in $\mu \mathrm{V}$ ) was measured as the difference between baseline and the negative peak. Peripheral neuropathy was excluded by determining the nerve conduction velocity and $\mathrm{F}$ waves of the tibial and peroneal nerves. No patient with any signs of neuropathy was included.

\section{Statistical analysis}

Basic data for patients and controls were compared using the Mann-Whitney $U$ test and $\chi^{2}$ test wherever appropriate. Patients were divided into two subgroups according to their UHDRS score: those with a UHDRS score $<25$ points $(n=10)$ formed the E-HD group while the remainder formed the M-HD group $(n=10)$.

Because the HRV indices were skewed, all HRV indices (except the LF/HF ratio) were log transformed; after logarithmic transformation, the HRV indices showed a normal distribution. As most of the HRV variables are strongly age dependent, a multivariate analysis was used with group (controls, E-HD, M-HD) as an independent factor, the HRV indices as dependent variables, and age as a covariate. The statistical significances of group differences were re-evaluated using a one way analysis of variance with post hoc comparisons using Duncan's multiple range test. Correlations between variables were calculated using Pearson correlation coefficients.

Standard normal values for the HRV indices were computed from the measured values of 60 healthy controls. HRV indices below the 2.3 centile of the measured values of healthy controls were considered pathological. ${ }^{1019}$ We used our standard normal values as normal values for the SSR; pathological latencies were defined as latencies 3 SDs higher than those of normal people; pathological amplitudes were $\leqslant 260$ $\mu \mathrm{V}$ at the hands and $\leqslant 240 \mu \mathrm{V}$ at the feet. ${ }^{18} \mathrm{~A}$ fall in systolic (diastolic) blood pressure of more than 20 (10) $\mathrm{mm} \mathrm{Hg}$ on standing was considered to be abnormal. ${ }^{20}$

\section{RESULTS}

\section{Clinical data and nerve conduction velocity}

For all patients, both the sensory and motor nerve conduction velocity were normal (data not shown). Patients of the E-HD group had a lower mean (SD) UHDRS score $(\mathrm{p}<0.001)$ : 14.6 (7.9) points (range 5-24) v $37.2(5.3)$ points (range 30-45). Moreover, in the E-HD group, the disease had a significantly shorter duration $(2.3$ (1.6) $v 4.7$ (2.2) years; $\mathrm{p}<0.05)$. No significant differences were found in the basic variables (age, sex, smoking) between the two patient subgroups, and when each of the two subgroups was compared with healthy controls. Three patients each from the E-HD and M-HD groups had received neuroleptics before the start of the study; these were discontinued three to eight days before the start of the study.

Three patients $(16 \%)$ reported subjective complaints suggestive of autonomic dysfunction, including sexual dysfunction $(n=2)$, excessive sweating $(n=1)$, and dizziness accompanied by a decrease in orthostatic blood pressure $(n=1)$.

\section{HRV (five minute resting and deep breathing test)}

To guarantee a standardised and comparably high quality for all HRV studies, only patients showing at least $98 \%$ correctly registered artifact-free QRS complexes during the examination period were included in the statistical analysis. For two patients with HD, statistical evaluation of the HRV study was not possible because of movement related ECG artifacts, and they were therefore excluded from the subsequent evaluation.

The multivariate analysis (multivariate analysis of covariances df 2;74) showed a significant effect for the factor "group" (controls, E-HD, M-HD) on the coefficient of variation $(\mathrm{CVr})$ at rest $(F=4.23, \mathrm{p}=0.018)$ and the spectral $\mathrm{HF}$ power $(F=4.32, \mathrm{p}=0.017)$, as well as a clear trend for the LF/HF ratio $(F=2.89, \mathrm{p}=0.062)$. The mean HF power and the CVr in the M-HD group were significantly lower not just in comparison with healthy probands, but also compared with the E-HD group (analysis of variance with Duncan's multiple range test, $\mathrm{p}<0.05)$. Although the CVr is dependent on resting heart rate, ${ }^{10}$ the significant group effects were still maintained when, in addition to age, the resting heart rate was considered as a second covariate.

Table 2 shows the mean values of the HRV indices of the patient subgroups and controls. Patients of the M-HD group showed the lowest HRV indices; significant differences compared with healthy people resulted for the variation coefficients both under resting conditions (CVr) and during deep respiration $(\mathrm{CVd})$, the root mean square of successive differences (RMSSDr), the spectral HF power, the mean circular resultant, and the LF/HF ratio. Except for the LF/HF ratio, which describes the sympathovagal balance, the remaining HRV indices mentioned mainly reflected modulation of vagal activity to the heart rate. Overall, therefore, the pattern of findings illustrates that vagal modulation is lowered under both conditions - that is, at rest and during deep breathing -in patients with medium stage HD compared with healthy people. The reduced modulation of vagal activity is accompanied by a subsequent displacement of the sympathovagal balance in favour of sympathetic modulation (expressed as a higher LF/HF ratio in patients of the M-HD group than patients of the E-HD group).

There was a significantly negative correlation between the severity of clinical HD symptoms (measured by the motor subscore of the UHDRS) and the vagal HRV indices; the highest correlation (fig 1) was between the HF power and the UHDRS $(r=-0.52, p=0.028)$. No correlation existed between the vagal HRV indices and the CAG repeats or the duration of the disease. 
Table 2 Heart rate variability (five minute resting study and deep breathing test) in healthy controls and in patients with Huntington's disease

\begin{tabular}{|c|c|c|c|c|}
\hline & $\begin{array}{l}\text { Controls } \\
(n=60)\end{array}$ & $\begin{array}{l}\text { Patients (total) } \\
(n=18)\end{array}$ & $\begin{array}{l}\text { E-HD group } \\
(n=9)\end{array}$ & $\begin{array}{l}\text { M-HD group } \\
(n=9)\end{array}$ \\
\hline \multicolumn{5}{|l|}{5 min resting study } \\
\hline $\mathrm{HRr}$ (beats/min) & $73.1(11.1)$ & 78.7 (12.0) & $78.2(10.5)$ & 79.2 (13.9) \\
\hline$C \operatorname{Cr}(\%)$ & $0.65(0.19)$ & $0.58(0.21)$ & $0.69(0.15)$ & $0.46(0.20)^{*} \dagger$ \\
\hline RMSSDr (ms) & $1.43(0.28)$ & $1.32(0.35)$ & $1.44(0.30)$ & $1.20(0.38)^{*}$ \\
\hline LF power $\left(\mathrm{ms}^{2}\right)$ & $2.87(0.45)$ & $2.88(0.41)$ & $3.08(0.32)$ & $2.68(0.39)$ \\
\hline HF power $\left(\mathrm{ms}^{2}\right)$ & $2.66(0.46)$ & $2.53(0.64)$ & $2.84(0.35)$ & $2.20(0.71)^{*} \dagger$ \\
\hline LF/HF ratio & 2.33 (2.19) & $3.15(2.45)$ & 2.07 (1.09) & $4.23(2.97)^{*}$ \\
\hline \multicolumn{5}{|l|}{ Deep breathing test } \\
\hline $\mathrm{CVd}(\%)$ & $0.88(0.19)$ & $0.83(0.19)$ & $0.92(0.09)$ & $0.74(0.22)^{*}$ \\
\hline RMSSDd (ms) & $1.58(0.24)$ & $1.51(0.25)$ & $1.60(0.15)$ & $1.42(0.30)$ \\
\hline MCR & $-1.38(0.25)$ & $-1.51(0.28)$ & $-1.42(0.22)$ & $-1.60(0.32)^{*}$ \\
\hline \multicolumn{5}{|c|}{$\begin{array}{l}\text { All HRV indices (except the LF/HF ratio) were log transformed. The values given are means (SD). } \\
\text { E-HD, Early stage Huntington's disease; M-HD, middle stage Huntington's disease. HRV, heart rate } \\
\text { variability; HRr, resting heart rate; CVr, CVd, coefficient of variation; RMSSDr, RMSSDd, root mean square } \\
\text { of successive differences; MCR, mean circular resultant; LF, low frequency; HF, high frequency. } \\
\text { Statistical comparison was by one way analysis of variance with post hoc comparison of means by Duncan's } \\
\text { multiple range test: * } p<0.05 \text { compared with healthy controls; } \dagger p<0.05 \text { compared with the E-HD group. }\end{array}$} \\
\hline
\end{tabular}

There were at least two pathologically lowered HRV indices ( $\leqslant 2.3$ centiles of the values of healthy controls) in either the resting or deep breathing studies for three of the patients. In two of these, at least three HRV indices of both tests, the resting and deep breathing study, were lowered abnormally, which we interpreted as a definite sign of the presence of parasympathetic dysfunction. A 30 year old man and a 54 year old woman of the M-HD group were affected.

\section{Blood pressure response to standing up and SSR}

Both tests allow inferences to be made about the function of the sympathetic nervous system. Under resting conditions, all patients had normal blood pressure. The average differences in systolic and diastolic blood pressure both immediately after standing up and one minute later were not significantly different between the E-HD and M-HD groups (data not shown); also, there was no significant correlation between the blood pressure differences and the motor subscore of the UHDRS, the CAG repeats, or the duration of the disease. A 30 year old patient of the M-HD group showed a pathological reduction in blood pressure (systolic and diastolic); in the same patient there was a bilateral, pathological latency delay in the SSRs of the hands, and there were signs of a parasympathetic dysfunction (reduction of at least three HRV indices during both the resting and deep breathing studies).

The SSRs could not be evaluated in five patients because of artifacts. Table 3 shows the average latencies and amplitudes of the SSRs of the hands and feet for each side of the body in the remaining 15 patients. The respective values for the right and left sides correlated significantly with one another (latencies at the feet and hands: $r=0.89-0.90, \mathrm{p}<0.0001$; amplitudes

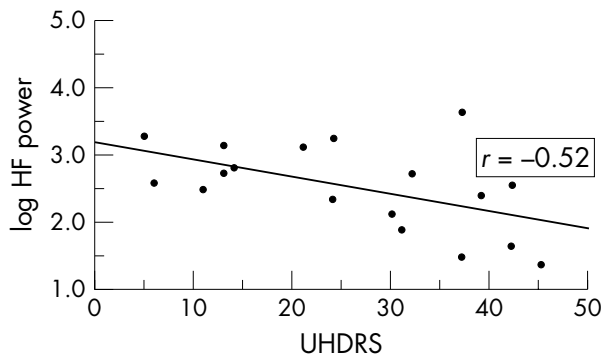

Figure 1 Relation between the severity of clinical symptoms of Huntington's disease (measured by the motor subscore of the unified Huntington's disease rating scale (UHDRS)) and the vagally mediated high frequency (HF) power. at the feet and hands: $r=0.88-0.93, \mathrm{p}<0.0001)$; no pathological body asymmetry existed. Compared with patients of the E-HD group, those in the M-HD group tended to show smaller SSR amplitudes at the hands (multiple analysis of covariance 1;12 DF, $F=3.79, \mathrm{p}=0.076$ ), but not at the feet. The mean SSR latencies did not differ between the groups. A marginal correlation between the UHDRS score and the SSR amplitudes was found at the hands (right: $r=-0.48$; left: $\mathrm{r}=-0.53$; $\mathrm{p}<0.08$ ). Neither the duration of the disease nor the CAG repeats correlated with the SSR latencies or amplitudes.

Three patients showed pathological SSR parameters: in one case there was a latency prolongation in the SSRs of the hands, while two more cases showed a pathological reduction of amplitude in the SSRs of the feet $(\leqslant 240 \mu \mathrm{V})$. All three patients belonged to the M-HD group.

\section{Combination of the results of all three autonomic tests} In total, four of 18 patients (22\%) showed pathological findings in one of the autonomic tests used (HRV at rest and during deep respiration, SSRs, orthostatic blood pressure test). Only patients of the M-HD group were affected. In this group, the proportion of patients with autonomic dysfunction (44\%) was significantly larger than in the E-HD group (one tailed $\chi^{2}$ tests, $\mathrm{p}<0.05$ ). Only one of these four patients for which the presence of an autonomic dysfunction could be confirmed

Table 3 Sympathetic skin responses (SSRs) in patients with Huntington's disease

\begin{tabular}{|c|c|c|}
\hline & E-HD group $(n=7)$ & $M-H D$ group $(n=8)$ \\
\hline \multicolumn{3}{|l|}{$\begin{array}{l}\text { SSRs palms } \\
\text { Latencies (ms) }\end{array}$} \\
\hline $\begin{array}{l}\text { Right side } \\
\text { Left side }\end{array}$ & $\begin{array}{l}1.57(0.13)(1.52) \\
1.59(0.13)(1.60)\end{array}$ & $\begin{array}{l}1.61(0.20)(1.57) \\
1.60(0.220(1.57)\end{array}$ \\
\hline \multicolumn{3}{|l|}{ Amplitudes $(\mu \mathrm{V})$} \\
\hline $\begin{array}{l}\text { Right side } \\
\text { Left side }\end{array}$ & $\begin{array}{l}2399(1521)(2130) \\
2284(1626)(1910)\end{array}$ & $\begin{array}{l}1095(637)(1034) \\
1014(657)(866)\end{array}$ \\
\hline \multicolumn{3}{|l|}{ SSRs feet } \\
\hline \multicolumn{3}{|l|}{ Latencies (ms) } \\
\hline Right side & $2.24(0.15)(2.22)$ & $2.17(0.26)(2.09)$ \\
\hline Left side & $2.23(0.15)(2.22)$ & $2.18(0.23)(2.19)$ \\
\hline \multicolumn{3}{|l|}{ Amplitudes $(\mu \mathrm{V})$} \\
\hline $\begin{array}{l}\text { Right side } \\
\text { Left side }\end{array}$ & $\begin{array}{l}925(515)(741) \\
1037(531)(718)\end{array}$ & $\begin{array}{l}864(658)(720) \\
1017(651)(825)\end{array}$ \\
\hline
\end{tabular}

The values given are means (SD) (median) E-HD, Early stage Huntington's disease; M-HD, middle stage Huntington's disease. 
reported subjective complaints in their medical history that suggested autonomic dysfunction.

\section{DISCUSSION}

The study presented was designed to clarify whether HD is associated with disrupted function of the ANS. The main findings of our study were that $(a)$ autonomic dysfunction can already be confirmed in about $44 \%$ of patients at a relatively early stage of HD (25-45 points on the motor subscore of the UHDRS), whereby both the sympathetic and parasympathetic system are affected, and $(b)$ an inverse association exists between the severity of clinical symptoms of HD (measured by the motor subscore of the UHDRS) and the modulation of neurocardiac vagal activity - that is, a high UHDRS motor score is associated with a low vagal modulation and vice versa.

In the largest study until now on this subject, Sharma and colleagues $^{5}$ examined ANS function in 22 patients with HD using classical HRV bedside tests as well as SSRs. They also verified the existence of both sympathetic and parasympathetic dysfunction in patients with HD. It is not clear in their study whether patients with a UHDRS motor subscore greater than 25 points were exclusively affected (as we found). Because of the lack of correlation of the HRV indices between the resting study and the Valsalva manoeuvre, Sharma and colleagues concluded that autonomic neurocardiac regulation in patients with HD is characterised by an imbalance between the parasympathetic and sympathetic control of the heart. ${ }^{5}$ Interestingly, ANS dysfunction during major depression is characterised by a very similar pattern of findings, and, indeed, depressive patients in the Valsalva experiment showed functional disturbances in the interaction between sympathetic and parasympathetic control of the heart.' As Sharma and colleagues also included depressive patients ( $36 \%$ of their patients were depressed), they presumably could not determine whether the autonomic imbalance they found in patients with HD was due to HD, the depression, or a combination of both factors. In our study, clinically non-depressive patients with HD with a UHDRS motor subscore of at least 25 points showed lower vagal HRV indices and a subsequently higher $\mathrm{LF} / \mathrm{HF}$ ratio than healthy controls. If one accepts the LF/HF ratio as an index for sympathovagal balance, ${ }^{13-15}$ our findings suggest that modulation of cardiac vagal activity is reduced in patients with $\mathrm{HD}$, so that sympathetic modulation starts to predominate.

Apart from depression, the psychiatric symptom "anxiety" may also influence the measured HRV, even though studies so far on the influence of anxiety symptoms or panic on HRV have been contradictory: compared with healthy people, patients with anxiety or panic symptoms show reduced sympathetic nerve activity leading to a relative predominance of vagal tone, ${ }^{21}$ or show no difference in autonomic reactivity, whereas another series of well designed studies has even produced evidence for decreased cardiovagal and relatively raised adrenergic reactivity in patients with panic disorder ${ }^{23-25}$ Even though we cannot definitively exclude the possibility that "anxiety" influenced ANS function in our patients with HD, we consider it to be unlikely for the following reasons. (a) All patients were examined by an experienced psychiatrist (MA), and in none of our patients were diagnostic criteria (DSM-III-R) fulfilled for the presence of an anxiety disorder. (b) As the means of the HRV indices (table 2) illustrate, patients in the E-HD group did not differ significantly from healthy controls, and indeed most HRV indices were very similar in the two groups. If the factor "anxiety" had systematically reduced the measured HRV (cardiovagal modulation), then clearly this only happened in patients of the M-HD group. We consider this unlikely, especially as the conditions during measurement were the same for all patients and controls; all HRV measurements were always measured at the same location by the same investigator (TS). (c) Yeragani and colleagues $^{25}$ found no correlation between the anxiety state of the patients and any of the HRV measures, which contradicts a direct association between anxiety symptoms and the measured HRV. Moreover, under resting conditions, patients with panic disorder did not differ significantly from healthy controls with regard to the absolute mid-frequency power (0.07-0.15 Hz; this frequency corresponds to the LF power defined as $0.04-0.15 \mathrm{~Hz}$ in our study), the HF power $(0.2-0.5$ $\mathrm{Hz}$; this corresponds to the HF power defined as $0.15-0.4 \mathrm{~Hz}$ in our study), and also the LF/HF ratio. In our study, patients in the M-HD group showed reduced parasympathetic indices (CVr, HF power) and a higher LF/HF ratio not only during deep respiration, but also under resting conditions compared with controls. This pattern of findings supports our hypothesis that, in patients with HD, the extent of autonomic dysregulation exceeds the (clearly only latent) dysregulation described for patients with panic disorder.

Compared with the study of Sharma and colleagues, ${ }^{5}$ we found pathological SSR findings far less often. As a comparison of the mean motor UHDRS subscores illustrates, the patients with HD in the study of Sharma et al were more severely afflicted with the disease than those in our study (36.8 (5.6) points $v 26.4$ (13.0) points). In our study, which only investigated patients in early and middle stages of HD, we did not identify any correlation between the motor UHDRS subscale and measurements of sympathetic nerve function (measured by orthostatic blood pressure regulation and the SSRs). However, there was a linear inverse correlation between the motor UHDRS subscale and the modulation of cardiac vagal activity (measured as the HRV). This pattern of findings strongly suggests that a continuous decrease in vagal modulation occurs during the course of $\mathrm{HD}$, a potentially important and clinically relevant finding, which needs to be verified in future longitudinal studies. Current concepts of cardiovascular disease state that a raised sympathetic activity is related to a higher susceptibility to life threatening cardiac arrhythmias and the development of coronary heart disease, ${ }^{26}$ and that, conversely, vagal activity exerts a cardioprotective effect. ${ }^{27}{ }^{28} \mathrm{~A}$ reduction in cardiac vagal modulation (measured as a reduction in HRV) is associated with increased cardiovascular morbidity and mortality: such an association has been shown for a number of diseases including alcoholism, diabetes mellitus, and myocardial infarction. ${ }^{29-33}$ Moreover, an altered autonomic balance (as indicated by an attenuation of vagal modulation) seems to contribute to sudden cardiac death even in apparently healthy subjects. ${ }^{34}$ In the light of this, we consider it probable that the observed pattern of findings, consisting of reduced modulation of cardiac vagal activity and subsequent displacement of the sympathovagal balance in favour of sympathetic activity, is at least partially responsible for the relatively high risk of cardiovascular morbidity and mortality in patients with HD; Haines and Conneally ${ }^{35}$ examined death certificates (which can provide, although incompletely, information on the causes of death) for 252 patients with HD and found that pneumonia and cardiovascular disease were the commonest causes of death, both as primary and contributory factors, accounting for $42 \%$ and $33 \%$ respectively, whereas no other cause (excluding HD itself) reached $10 \%$.

Evaluating the patient's history alone is insufficient to determine whether ANS dysfunction exists in patients early in the course of HD; autonomic functional tests must also be performed. Indeed, only one of the four patients with HD who had confirmed autonomic dysfunction reported corresponding subjective complaints suggestive of ANS dysfunction in our study. This observation is consistent with the idea that degeneration of ANS must be profound before clinical signs start to appear. ${ }^{36}$ We can only speculate on the topography of the neuronal damage responsible for ANS dysfunction in HD. In principle, one has to consider that ANS dysfunction may also be the result of a defective end organ function. Although we excluded patients with HD who had pre-existing cardiac disease, a subclinical cardiomyopathy could have remained 
undetected in individual cases despite normal thoracic radiographic, ECG, or echocardiographic findings. Defective end organ function can be caused on the one hand by damage to the cardiac muscle tissue itself, or on the other by damage to intramurally located, small nerve fibres. Investigations of the ANS in patients with HD who have a subclinical, HD associated cardiomyopathy have not yet been performed. In patients with congestive dilatative cardiomyopathy, disturbances in cardiovagal function can already be shown at early (before heart failure) stages of the disease. ${ }^{37}$ However, we consider it very unlikely that cardiomyopathy alone is responsible for the vagal dysfunction in patients with HD; on the one hand, we found not only cardiovagal, but also sympathetic, ANS dysfunction, while on the other a correlation was observed between the severity of clinical HD symptoms (measured by the motor UHDRS subscale) and the modulation of cardiac vagal activity, thus allowing a direct association between HD and ANS dysregulation to be assumed. Previous studies have postulated HD associated disorders in the central autonomic network-for example, the hypothalamus and its connection to the neocortex, the limbic system, brainstem, and spinal cord..$^{35}$ A pupillometric study in patients with HD suggests neuronal damage in the area of the Edinger-Westphal nucleus. ${ }^{3}$ Neuropathological studies have shown degeneration in the area of the dorsal vagal nucleus and destruction of vegetative neurones in the pre-optical section of the hypothalamus. ${ }^{38}$ Damage to hypothalamic cells could indeed explain unusual features in various parasympathetic and sympathetic centres.

To our knowledge, this is the first study of ANS function in patients with genetically confirmed HD at early and middle stages of the disease. In patients with middle stage HD (UHDRS 25-50 points), we found evidence of reduced modulation of cardiovagal activity with a subsequent predominance of sympathetic activity modulation. This study focuses primarily on standardised measurements of HRV. Further studies to clarify ANS function in HD are required which should examine in more detail the vascular regulation, especially the interplay of vagal and sympathetic modulation, in patients with HD-for example, with regard to the baroreflex.

\section{ACKNOWLEDGEMENTS}

We thank Dr J P Keogh for assistance with translating the manuscript, $\mathrm{T}$ Majewski (Department of Internal Medicine) for electrocardiographic evaluations, and H R Bingöl (Department of Neurology) for technical support.

\section{Authors' affiliations}

J Andrich, C Saft, T Postert, P Kraus, H Przuntek, Department of Neurology, St Joseph Hospital, Ruhr-University Bochum, Germany

T Schmitz, M W Agelink, Department of Biological Psychiatry and Neuroscience, EvK Gelsenkirchen, Ruhr-University Bochum

J T Epplen, Department of Human Genetics, Ruhr-University Bochum

\section{REFERENCES}

1 Lodi R, Shapira T, Manners D, et al. Abnormal in vivo skeletal muscle energy metabolism in Huntington's Disease and Dentatopallidoluysian Atrophy. Mov Disord 2000;15:241

2 Aminoff MJ, Gross M. Vasoregulatory activity in patients with Huntington's chorea. J Neurol Sci 1974;21:33-8.

3 Den Heijer JC, Bollen W, Reulen JPH, et al. Autonomic nervous function in Huntington's Disease. Arch Neurol 1988;45:309-12.

4 Camerlingo $M$, Bottacchi E, Gambaro $P$, et al. Parasympathetic function in Huntington's Disease. Functional Neurology 1987;2:227-20.

5 Sharma KR, Romano JG, Ram Ayyar D, et al. Sympathetic skin response and heart rate variability in patients with Huntington's Disease. Arch Neurol 1999;56:1248-52.

6 Task Force of the European Society of Cardiology and the North American Society of Pacing and Electrophysiology. Heart rate variability. Standards of measurement, physiological interpretation, and clinical use. Circulation 1996;93:1043-65.

7 Low PA, Pfeifer MA. Standardization of clinical tests for practice and clinical trials. In: Low PA, ed. Clinical autonomic disorders: evaluation and management. Boston: Little Brown and Co, 1993.

8 Unified Huntington's Disease Rating Scale: reliability and consistency. Huntington Study Group. Mov Disord 1996;11:136-42.
9 Agelink MW, Majewski T, Wurthmann C, et al. Autonomic neurocardiac function in patients with major depression and effects of antidepressive treatment with nefazodone. J Affect Disord 2001;62:187-98.

10 Agelink MW, Malessa R, Baumann B, et al. Standardized tests of heart rate variability (HRV): normal ranges obtained from 309 healthy humans and effects of age, gender and heart rate. Clin Auton Res 2001;11:99-108.

11 Sinnreich R, Kark JD, Friedlander $Y$, et al. Five minute recordings of heart rate variability for population studies: repeatability and age-sex characteristics. Heart 1998:80:156-62.

12 Berger RD, Akselrod S, Gordon D, et al. An efficient algorithm for spectral analysis of heart rate variability. IEEE Trans Biomed Eng 1986;33:900-4

13 Pagani M, Lombardi F, Guzzetti S, et al. Power spectral analysis of heart rate and arterial pressure variabilities as a marker of sympathovagal interaction in man and conscious dog. Circ Res 1986;59:178-93.

14 Lanza GA, Pedrotti P, Rebuzzi AG, et al. Usefulness of the addition of heart rate variability to Holter monitoring in predicting in-hospital cardiac events in patients with unstable angina pectoris. Am J Cardiol 1997:80:263-7.

15 Urbina EM, Bao W, Pickoff AS, et al. Ethnic (black-white) contrasts in heart rate variability during cardiovascular reactivity testing in male adolescents with high and low blood pressure: the Bogalusa Heart Study. Am J Hypertens 1998;11:196-202.

16 Eckberg DL. Sympathovagal balance: a critical appraisal. Circulation 1997; 96:3224-32

17 Weinberg CR, Pfeiffer MA. An improved method of measuring heart rate variability: assessment of cardiac autonomic function. Biometrics 1984:40:855-61

18 Schwalen S, Jörg J. Peripher autonome Potentiale: Normwerte unter verschiedenen Bedingungen. Zeitschrift EEG-EMG 1993;24:242-6.

19 Ziegler D, Laux G, Dannehel K, et al. Assessment of cardiovascular autonomic function: age-related normal ranges and reproducibility of spectral analysis, vector analysis and standard tests of heart rate variation and blood pressure response. Diabet Med 1992;9:166-75.

20 Ewing DJ. Analysis of heart rate variability and other noninvasive tests with special reference to diabetes mellitus. In: Bannister R, Mathias CJ, eds. Autonomic failure: a textbook of clinical disorders of autonomic system. Oxford: Oxford University Press, 1992:312-33.

21 McCraty R, Atkinson M, Tomasino D, et al. Analysis of twenty-four hour heart rate variability in patients with panic disorder. Biol Psychiatry $2001 ; 56: 131-50$

22 Stein MB, Asmundson GJG. Autonomic function in panic disorder: cardiorespiratory and plasma catecholamine responsivity to multiple challenges of the autonomic nervous system. Biol Psychiatry 1994;36:548-58

23 Yeragani VK, Meiri PC, Pohl R, et al. Heart rate and blood pressure changes during postural change and isometric handgrip exercise in patients with panic disorder and normal controls. Acta Psychiatr Scand 1990;81:9-13.

24 Yeragani VK, Balon R, Pohl R, et al. Decreased R-R variance in panic disorder patients. Acta Psychiatr Scand 1990;81:554-9.

25 Yeragani VK, Pohl R, Berger R, et al. Decreased heart rate variability in panic disorder patients: a study of power spectral analysis of heart rate. Psychiatry Res 1993;46:89-103.

26 Schwartz PJ, La Rovere MT, Vanoli E. Autonomic nervous system and sudden cardiac death. Experimental basis and clinical observations for post myocardial infarction risk stratification. Circulation 1992:85:177-91.

27 Lown B, Verrier RL. Neural activity and ventricular fibrillation. N Engl J Med 1976;294:1165-70

28 Ben-David J, Zipes DP. Autonomic neural modulation of cardiac rhythm part I. Basic concepts. Modern Concepts of Cardiovascular Disease 1988;57:41-6.

29 Johnson RH, Robinson BJ. Mortality in alcoholics with autonomic neuro-pathy. J Neurol Neurosurg Psychiatry 1988;51:476-80.

30 O'Brien IA, McFadden JP, Corall JM. The influence of autonomic neuropathy on mortality in insulin-dependent diabetes. QIM 1991;290:495-502.

31 La Rovere MT, Bigger JT, Marcus Fl, et al. Baroreflex sensitivity and heart rate variability in prediction of total cardiac mortality after myocardial infarction. ATRAMI (Autonomic Tone and Reflexes After Myo-cardial Infarction) Investigators. Lancet 1998;351:478-84.

32 Kleiger RE, Miller IP, Bigger JT, et al. Decreased heart rate variability and its association with increased mortality after acute myocardial infarction. Am J Cardiol 1987;59:256-62.

33 Ewing DJ, Clarke BF. Autonomic neuropathy: its diagnosis and prognosis. J Clin Endocrinol Metab 1986;15:855-89.

34 Molgaard H, Sorensen KE, Bjerregaard P. Attenuated 24-h heart rate variability in apparently healthy subjects, subsequently suffering sudden cardiac death. Clin Auton Res 1991;1:233-7.

35 Haines JL, Conneally PM. Causes of death in Huntington's disease as reported on death certificates. Genet Epidemiol 1986;3:417-23.

36 Low PA. Quantitation of autonomic functions. In : Dyck PJ, Thomas PK, Griffin JW, et al, eds. Peripheral neuropathy. Philadelphia: WB Saunders Co, 1993:728-45

37 Amorim DS, Dargie HJ, Heer K, et al. Is there autonomic impairment in congestive (dilated) cardiomyopathy? Lancet $1981 ; \mathrm{i}: 525-7$.

38 Bird ED. Neuroendocrine changes in Huntington's Disease. Adv Neurol 1997;23:291-7.

39 Lavin PJ, Bone I, Sheridan P. Studies of hypothalamic function in Huntington's chorea. J Neurol Neurosurg Psychiatry 1981;44:414-18. 